\title{
A $H$-Infinity Control for Path Tracking with Fuzzy Hyperbolic Tangent Model
}

\author{
Guangsi Shi, Jue Yang, Xuan Zhao, Yanfeng Li, Yalun Zhao, and Jian Li \\ School of Mechanical Engineering, University of Science \& Technology Beijing, Beijing 100083, China \\ Correspondence should be addressed to Jue Yang; yangjue@ustb.edu.cn
}

Received 6 May 2016; Revised 3 September 2016; Accepted 5 October 2016

Academic Editor: Mario Russo

Copyright (C) 2016 Guangsi Shi et al. This is an open access article distributed under the Creative Commons Attribution License, which permits unrestricted use, distribution, and reproduction in any medium, provided the original work is properly cited.

\begin{abstract}
To achieve the goal of driver-less underground mining truck, a fuzzy hyperbolic tangent model is established for path tracking on an underground articulated mining truck. Firstly, the sample data of parameters are collected by the driver controlling articulated vehicle at a speed of $3 \mathrm{~m} / \mathrm{s}$, including both the lateral position deviation and the variation of heading angle deviation. Then, according to the improved adaptive BP neural network model and deriving formula of mediation rate of error estimator by the method of Cauchy robust, the weights are identified. Finally, $\mathrm{H}$-infinity control controller is designed to control steering angle. The results of hardware-in-the-loop simulation show that lateral position deviation, heading angle deviation, and steering angle of the vehicle can be controlled, respectively, at $0.024 \mathrm{~m}, 0.08 \mathrm{rad}$, and $0.21 \mathrm{rad}$. All the deviations are asymptotically stable, and error control is in less than $2 \%$. The method is demonstrated to be effective and reliable in path tracking for the underground vehicles.
\end{abstract}

\section{Introduction}

Articulated vehicle is widely used in underground mining. Researches on driver-less articulated vehicle have been carried out for many years to prompt efficiency and safety in underground mine. A key principle of autonomous driving in underground tunnel is to find a path for articulated vehicle to track at a reasonable high speed and avoiding crash into sidewall. Many automatic control algorithms can be applied in this filed.

In the field of fuzzy model, Zeng and Singh [1] and Wang [2] have theorized a fuzzy relational model, and its purpose is to build a fuzzy model to approximate the ideal control behavior. This fuzzy relationship can be seen as a fuzzy mapping from input space to the output space. Its main disadvantage is that many important dynamic information systems are ignored during the modeling process, and it is difficult to obtain good performances of controller. Takagi and Sugeno $[3,4]$ have proposed a fuzzy model named T-S, aiming to construct a series of linear equations to express each subsystem, then to make a global model through the membership functions of these subsystems. Its main drawback is that the complexity of the structure itself to establish a model requires lots of work offline. Fuzzy hyperbolic tangent model is better than the above models $[5,6]$.

Path tracking problem is a very important issue in the course of unmanned field. The former studies are based on articulated vehicle kinematics, and many scholars have done a lot of researches. Nayl et al. [7], Lee and Yoo [8], Ridley and Corke [9], and Xuan et al. [10] derived kinematic models of articulated vehicle and the reference model errors of path, and they used method of model predictive control for tracking control with simulation. The work efficiency and safety are decided by the accuracy of path tracking. Path tracking control design based on articulated vehicle kinematic characteristics has no good adaptability in underground mining environment. Dynamics characteristics are significant for path tracking control design [11]. Literature [12] considers the influence of articulated vehicle dynamics on sideslip, but there would be a big deviation as they only consider one aspect and the lack of system modeling.

To solve these problems above and to deal with this multivariable, strong coupling, highly complex nonlinear dynamical systems of underground mining vehicles, we use fuzzy hyperbolic model and design a nonlinear quadratic controller through testing field experiments by hardware-in-the-loop 


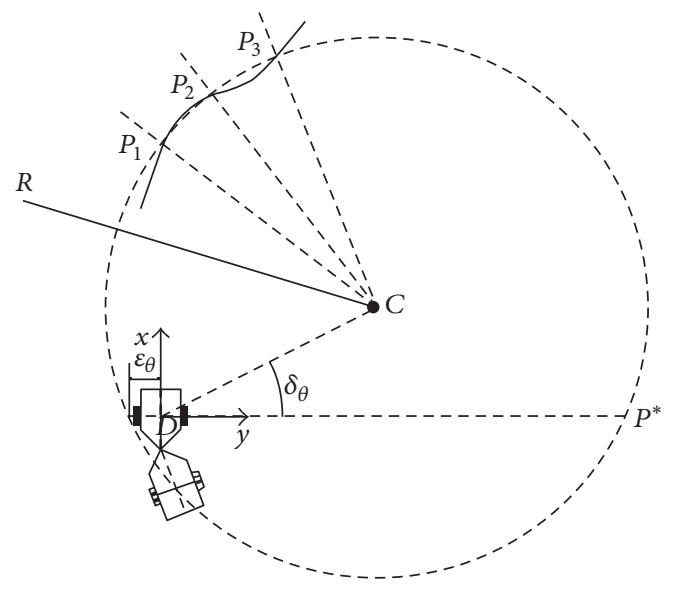

Figure 1: Trajectory curve and parametric model image of underground mining articulated vehicle.

(HIL) simulation to ensure the quality of control, aiming at achieving the goal of unmanned underground mining articulated vehicles. This method is to obtain kinematic relations of vehicle via the driver information, and the fact that driver repeatedly driving the process can make the relationship dynamics of the vehicle which are included in those data becomes increasingly evident. This method can also be considered to be intrinsically linked with a data mining to reduce the complexity of the modeling process, which greatly reduces the system error caused by the dynamics modeling. The coefficient matrix of fuzzy hyperbolic model nonlinear systems becomes constant matrix. The parameters of the identification via neural network, supervised learning methods, make the model more close to the real model. Also it is convenient to the control algorithm design. Young [13] has proposed variable structure controller for the first time, which was applied to robot control. Jafarov et al. [14] have proposed a new type of sliding PID controller. Although these methods can solve the control problem, they could not consider the robustness of the system.

$H$-infinity control is a good robustness design method, with clear design ideas, good control effect, and advantages, especially on model perturbation of multiple input multiple output (MIMO) systems. Finally, hardware verification control which results in the final loop (HIL) simulation is done in order to ensure quality control.

\section{Kinematic Model of Underground Articulated Vehicle}

2.1. Steering Deviation Model. Figure 1 shows the errors between the real and reference path. The circle centered by $C$ is the reference path. Ideally, the vehicle should pass $P_{1}, P_{2}$, and $P_{3}$. The variables are defined as follows $[5,15]$ :

(1) lateral displacement error: the lateral displacement error between the vehicle reference point and the corresponding point $P$ (the nearest point on the reference path);
(2) orientation error: the orientation error between the velocity orientation of $p$ and the tangential orientation of $P$;

(3) steering angle: relative rotation angle of underground mining articulated vehicle body before and after the horizontal plane.

These two variables can basically reflect the position and posture of articulated underground mining vehicles in the tunnel, and building the trajectory of articulated vehicles model can be achieved by the fuzzy hyperbolic method.

Figure 2(a) is the sketch of an artificial tunnel for testing of articulated vehicle driver-less control system. The truck used in this test is shown in Figure 2(b).

As can be seen in Figures 3-5, at speed of $3 \mathrm{~m}$ per second, by driver controlling the truck in the process of turning, the lateral deviation is stable at $1.5 \mathrm{~m}$. Heading angle deviation remains stable at around $0 \mathrm{rad}$, while body roll is obvious. The steering angle remains stable at $0.2 \mathrm{rad}$ with obvious adjustments. As shown in Figures 6 and 7, at speed of $3 \mathrm{~m}$ per second, rate of change of lateral deviation and the heading angle also is 0 , but there are more noise and severe jitter.

\subsection{Kinematic Models Based on Fuzzy Hyperbolic Model.} Near the origin, fuzzy hyperbolic model is a global model [6] with a global approximation performance. And it is not only an essentially nonlinear model, but also easy to express the dynamic characteristics of nonlinear systems. Therefore, according to this model, the controller can be designed so that the whole system can achieve the optimal performances. Compared with other fuzzy models, fuzzy hyperbolic model is more suitable for the control object known as multivariable nonlinear finite object.

Given a plant $n$ state variables number: $x=\left(x_{1}(t)\right.$, $\left.\ldots, x_{n}(t)\right)^{\mathrm{T}}$, and $p$ input variables numbers: $\mathbf{u}=\left(\mathbf{u}_{1}(t), \ldots\right.$, $\left.\mathbf{u}_{p}(t)\right)^{\mathrm{T}}$, and the fuzzy rules are used to describe this system by the following conditions, so this group of fuzzy rules is termed as the hyperbolic tangent type of fuzzy rules.

Fuzzy rules for the following forms:

$$
\begin{aligned}
& \text { IF } x^{1} \text { is } F_{x_{1}} \text { and } x^{2} \text { is } F_{x_{2}} \text { and } \ldots x^{n} \text { is } F_{x_{n}} \text { and } \mathbf{u}^{1} \text { is } F_{\mathbf{u}_{1}} \\
& \text { and } \mathbf{u}^{2} \text { is } F_{\mathbf{u}_{2}} \text { and } \ldots \mathbf{u}^{n} \text { is } F_{\mathbf{u}_{p}} \\
& \operatorname{THEN} \dot{x}_{l}= \pm c_{x_{1}} \pm c_{x_{2}} \cdots \pm c_{x_{n}} \pm c_{\mathbf{u}_{1}} \pm c_{\mathbf{u}_{2}} \cdots \pm c_{\mathbf{u}_{p}}(l= \\
& 1, \ldots, n)
\end{aligned}
$$

where $F_{x_{i}}(i=1, \ldots, n)$ and $F_{\mathbf{u}_{j}}(j=1, \ldots, p)$ are fuzzy subset including $(P)$ and $(N)$ corresponding to $x_{i}$ and $\mathbf{u}_{j}$ and $c_{x_{i}}(i=1, \ldots, n)$ and $c_{\mathbf{u}_{j}}(j=1, \ldots, p)$ are constant of $F_{x_{i}}$ and $F_{\mathbf{u}_{i}}(j=1, \ldots, p)$.

Let $\delta_{d}=x_{1}, \delta_{\theta}=x_{2}$, and $\mathbf{u}=\gamma$.

There are certain mathematical relationships between manned lateral deviation, heading angle deviation, the steering angle, and rate of change of the lateral deviation and heading angle deviation from the data observed from relevant papers [10]. And they can be roughly described as the following information through the professional experiences so as to 


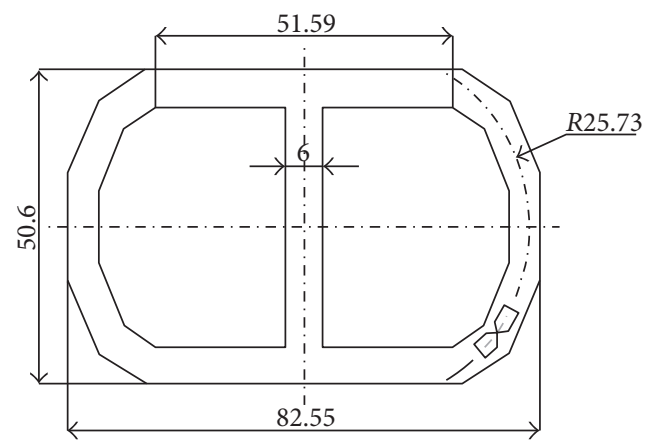

(a)

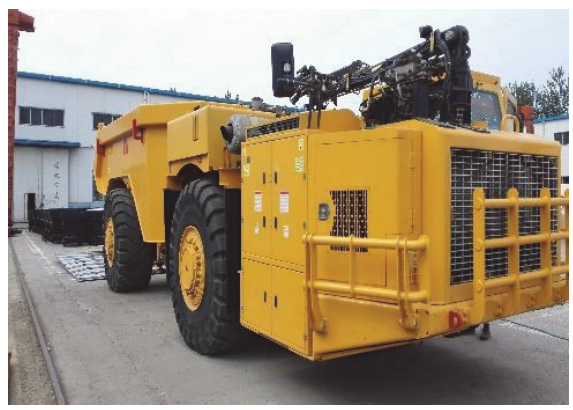

(b)

FIgURE 2: (a) The plan view of the tunnel. (b) The real truck.

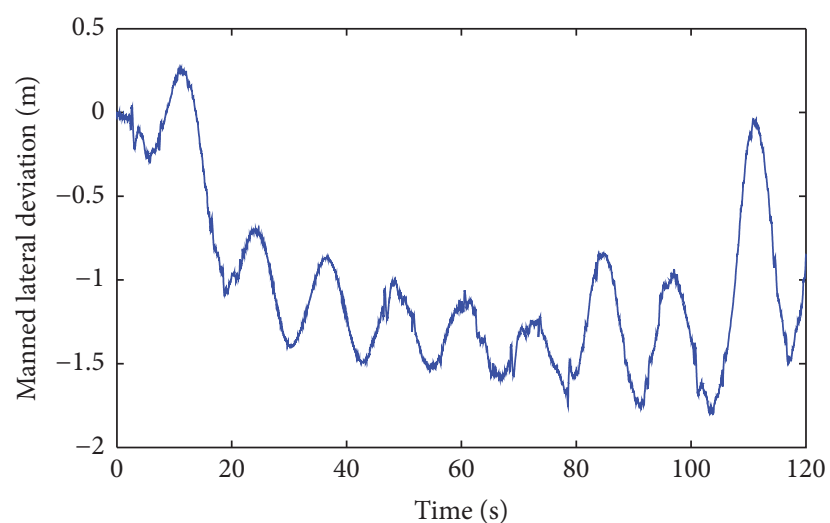

Figure 3: Manned lateral deviation.

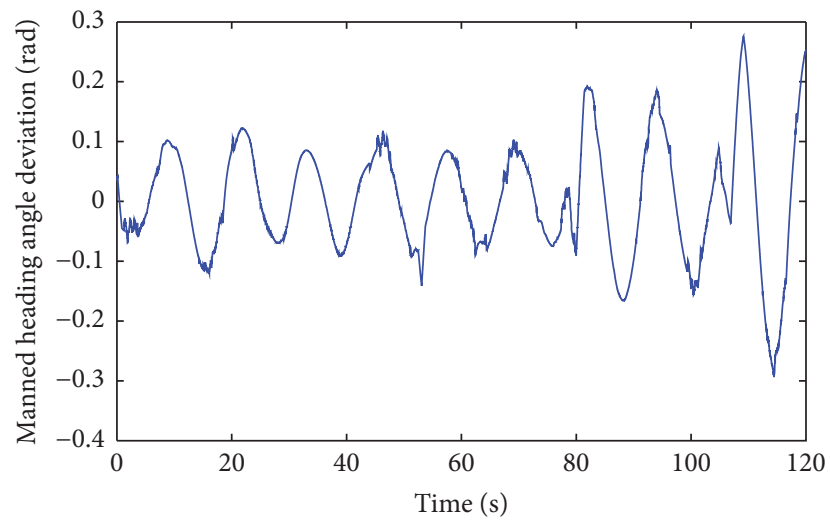

FIGURE 4: Manned heading angle deviation.

construct the corresponding fuzzy hyperbolic model of fuzzy rules:

$$
\begin{aligned}
& \mathrm{R}_{1}: \text { IF } x_{2} \text { is } P_{x_{2}} \text { THEN } \dot{x}_{1}=0.1 \\
& \mathrm{R}_{2}: \text { IF } x_{2} \text { is } N_{x_{2}} \text { THEN } \dot{x}_{1}=-0.1 \\
& \mathrm{R}_{3}: \text { IF } x_{1} \text { is } P_{x_{1}} \text { IF } x_{2} \text { is } P_{x_{2}} \text { IF } \mathbf{u} \text { is } P_{\mathbf{u}} \text { THEN } \dot{x}_{2}=0.01 \\
& \mathrm{R}_{4}: \text { IF } x_{1} \text { is } N_{x_{1}} \text { IF } x_{2} \text { is } P_{x_{2}} \text { IF } \mathbf{u} \text { is } P_{\mathbf{u}} \text { THEN } \dot{x}_{2}=0 \\
& \mathrm{R}_{5}: \text { IF } x_{1} \text { is } N_{x_{1}} \text { IF } x_{2} \text { is } N_{x_{2}} \text { IF } \mathbf{u} \text { is } P_{\mathbf{u}} \text { THEN } \dot{x}_{2}=0.08
\end{aligned}
$$

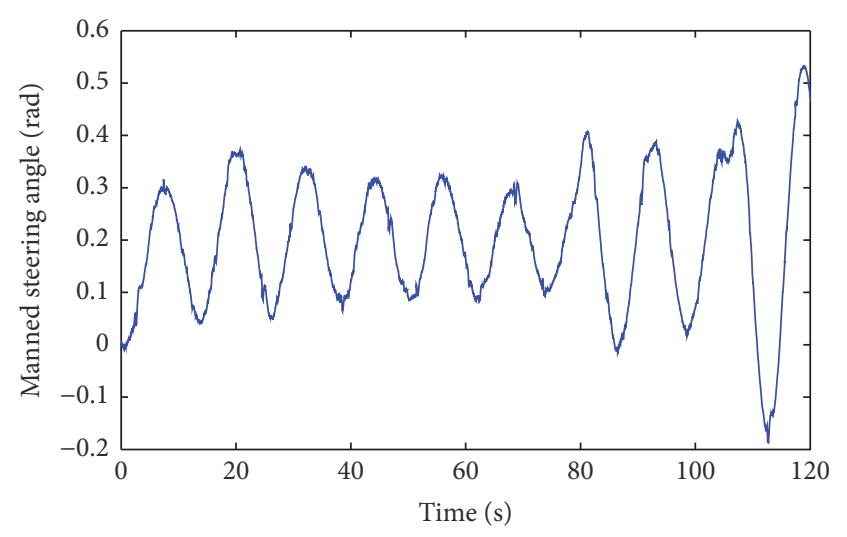

FIGURE 5: Manned steering angle.

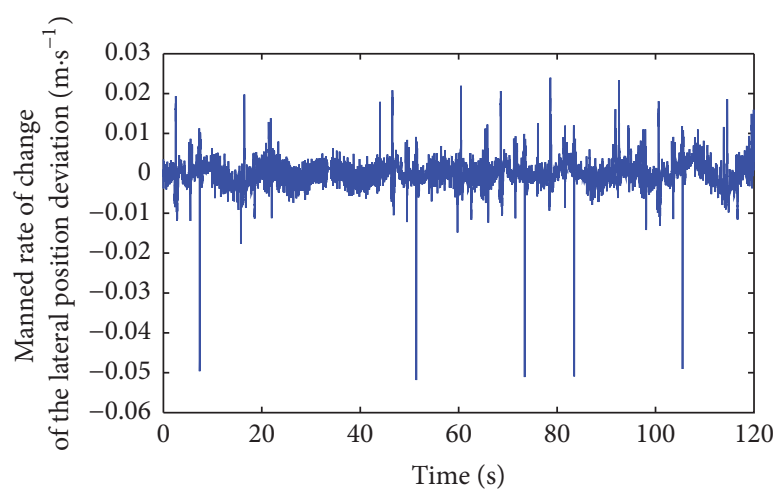

FIgURE 6: Manned rate of change of the lateral position deviation.

$\mathrm{R}_{6}:$ IF $x_{1}$ is $N_{x_{1}}$ IF $x_{2}$ is $N_{x_{2}}$ IF $\mathbf{u}$ is $N_{\mathbf{u}}$ THEN $\dot{x}_{2}=$ $-0.01$

$\mathrm{R}_{7}$ : IF $x_{1}$ is $P_{x_{1}}$ IF $x_{2}$ is $N_{x_{2}}$ IF $\mathbf{u}$ is $N_{\mathbf{u}}$ THEN $\dot{x}_{2}=0$

$\mathrm{R}_{8}$ : IF $x_{1}$ is $P_{x_{1}}$ IF $x_{2}$ is $P_{x_{2}}$ IF $\mathbf{u}$ is $N_{\mathbf{u}}$ THEN $\dot{x}_{2}=-0.08$

$\mathrm{R}_{9}$ : IF $x_{1}$ is $P_{x_{1}}$ IF $x_{2}$ is $N_{x_{2}}$ IF $\mathbf{u}$ is $P_{\mathbf{u}}$ THEN $\dot{x}_{2}=0.1$

$\mathrm{R}_{10}:$ IF $x_{1}$ is $N_{x_{1}}$ IF $x_{2}$ is $P_{x_{2}}$ IF $\mathbf{u}$ is $N_{\mathbf{u}}$ THEN $\dot{x}_{2}=-0.1$ 


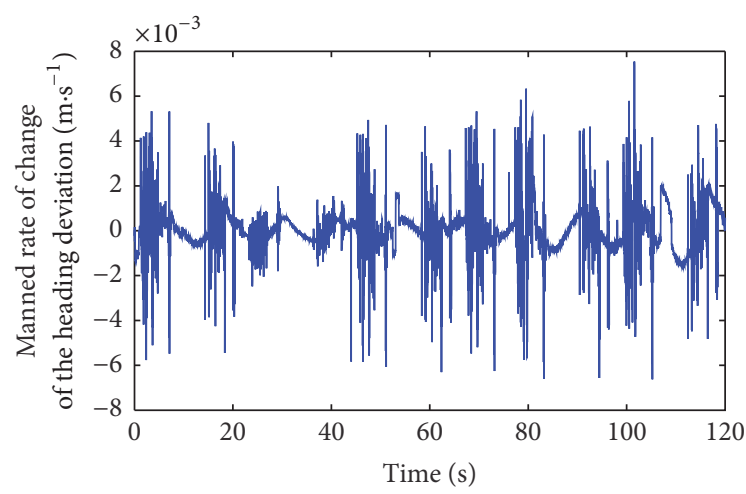

FIgURE 7: Manned rate of change of the heading deviation.

Given a set of fuzzy rules of hyperbolic tangent, define $P_{z}$ and $N_{z}$ (where $z$ are arbitrary state variables or input variables) as membership function (where $\mathbf{k}_{z}>0$ ):

$$
\begin{gathered}
P_{z}(x)=e^{-(1 / 2)\left(x-\mathbf{k}_{z}\right)}, \\
N_{z}(x)=e^{-(1 / 2)\left(x+\mathbf{k}_{z}\right)} .
\end{gathered}
$$

Let $\mathbf{k}_{x_{1}}=\mathbf{k}_{x_{2}}=\mathbf{k}_{\mathbf{u}}=1$.

Membership function:

$$
\begin{aligned}
& \mathbf{u}_{P_{x_{1}}}(x)=e^{-(1 / 2)(x-1)^{2}}, \\
& \mathbf{u}_{N_{x_{1}}}(x)=e^{-(1 / 2)(x+1)^{2}} \\
& \mathbf{u}_{P_{x_{2}}}(x)=e^{-(1 / 2)(x-1)^{2}}, \\
& \mathbf{u}_{N_{x_{2}}}(x)=e^{-(1 / 2)(x+1)^{2}} ; \\
& \mathbf{u}_{P_{\mathbf{u}}}(x)=e^{-(1 / 2)(x-1)^{2}}, \\
& \mathbf{u}_{P_{\mathbf{u}}}(x)=e^{-(1 / 2)(x+1)^{2}} .
\end{aligned}
$$

Then

$$
\dot{x}=\mathbf{A} \tanh \left(\mathbf{k}_{x} x\right)+\mathbf{B} \tanh \left(\mathbf{k}_{\mathbf{u}} \mathbf{u}\right),
$$

where

$$
\begin{aligned}
\mathbf{A} & =\left[\begin{array}{cc}
0 & 0.1 \\
0.01 & -0.1
\end{array}\right], \\
\mathbf{B} & =\left[\begin{array}{c}
0 \\
0.08
\end{array}\right], \\
\mathbf{k} & =\left[\begin{array}{cc}
1 & \\
1 & \\
& 1
\end{array}\right], \\
\tanh (x) & =\frac{e^{x}-e^{-x}}{e^{x}+e^{-x}}
\end{aligned}
$$

$$
\begin{aligned}
& \mathbf{k}_{x}=\operatorname{diag}\left(\mathbf{k}_{x_{1}}, \ldots, \mathbf{k}_{x_{n}}\right), \\
& \mathbf{k}_{\mathbf{u}}=\operatorname{diag}\left(\mathbf{k}_{x_{1}}, \ldots, \mathbf{k}_{x_{p}}\right) .
\end{aligned}
$$

Bku are linearized to Bu:

$$
\begin{aligned}
\dot{x} & =\mathbf{A} \tanh \left(\mathbf{k}_{x} x\right)+\mathbf{B u}, \\
{\left[\begin{array}{l}
\dot{x}_{1} \\
\dot{x}_{2}
\end{array}\right] } & =\left[\begin{array}{cc}
0 & 0.1 \\
0.01 & -0.1
\end{array}\right]\left[\begin{array}{l}
\tanh \left(x_{1}\right) \\
\tanh \left(x_{2}\right)
\end{array}\right]+\left[\begin{array}{c}
0 \\
0.08
\end{array}\right] \gamma .
\end{aligned}
$$

When the absolute value of $x$ is smaller, $\tanh (x) \approx x$, fuzzy hyperbolic model can be written as $\dot{x}=\mathbf{A} \tanh \left(\mathbf{k}_{x} x\right)+$ $\mathrm{Bu}$ that the system is a linear model when it is close to the equilibrium point.

\section{Parameter Identification Based on Improved Adaptive BP Neural Network}

Recognition technology evaluation includes two indicators. One is the identification accuracy, and the other is speed identification. Compared to other models in terms of fuzzy, topology of the neural network can be used to optimize the parameters for FHM [16]. The $A$ weights of mediation rate of adaptive BP neural network are deduced by Cauchy robust error, to eliminate the effect of outliers in the data and to fit the original data better.

Neural network topology is shown in Figure 8 of FHM. Controlled object state variables are $x=\left(x_{1}(t), \ldots, x_{n}(t)\right)^{\mathrm{T}}$ and input variables $\mathbf{u}=\left(\mathbf{u}_{1}(t), \ldots, \mathbf{u}_{m}(t)\right)^{\mathrm{T}}$ as the input of the neural network topology, and model output is the rate of change of the state variables $\mathbf{k}_{i}(i=1, \ldots, n), g_{j}(j=$ $1, \ldots, m), c_{i j}(i, j=1, \ldots, n)$, and $d_{i j}(i=1, \ldots, n, j=$ $1, \ldots, m)$ as the connection weights which need to be trained.

$\mathbf{A}$ and $\mathbf{B}$ are constant matrix, whose elements are the weights comprised of $c_{i j}$ and $d_{i j}$. If the hidden layer function $f_{1}(x)=\tanh (x)$ and output layer activation function $f_{2}(x)=$ $x$, then the following models can be obtained:

$$
\dot{x}=\mathbf{A} \tanh \left(\mathbf{k}_{x} x\right)+\mathbf{B} \tanh \left(\mathbf{k}_{\mathbf{u}} \mathbf{u}\right) .
$$

Obviously, when making u linearize, variables can be obtained

$$
\dot{x}=\mathbf{A} \tanh \left(\mathbf{k}_{x} x\right)+\mathbf{B u} .
$$

Thus, FHM may be built by the neural network model, because it can train FHM model parameters [6]. Since there are many jitters in the data of rate of change of horizontal and heading angle, the samples show a large number of outliers. So from the statistical view of robust, the traditional MSE aggravate the "outliers" of samples. Therefore, Cauchy robust error estimator can be used.

Let error of outputs be

$$
E=\frac{1}{2} \sum_{\mathbf{k}=1}^{q} \ln \left[1+\left(y_{\mathbf{k}}^{\prime}-y_{\mathbf{k}}\right)^{2}\right],
$$




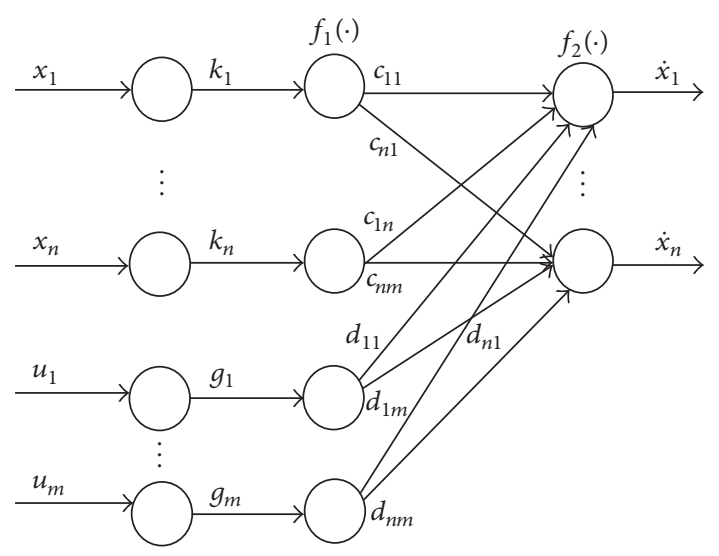

FIGURE 8: Fuzzy hyperbolic tangent model neural network topology.

where $y_{\mathbf{k}}^{\prime}$ is ideal output for the network and $y_{\mathbf{k}}$ is actual output.

Cauchy error estimator obviously does not depend on the initial weights and thresholds transition, and it can effectively eliminate the negative impact of "outliers," while retaining the main characteristics of the value of the output error and speeding up the convergence rate.

By the steepest descent method, it can obtain weights iterative equations of each layer of neurons:

$$
W(t+1)=W(t)-\mathbf{u} \Delta E
$$

where $\Delta E=\partial E / \partial \omega ; W=\left\{\omega_{i j}\right\}$.

So get a rate adjustment based on the following model parameters above $\mathrm{BP}$ algorithm:

$$
\begin{aligned}
c_{i j}(t+1)= & c_{i j}(t)+v \cdot \frac{y_{\mathbf{k}}^{\prime}-y_{\mathbf{k}}}{1+\left(y_{\mathbf{k}}^{\prime}-y_{\mathbf{k}}\right)^{2}} \cdot \tanh \left(\mathbf{k}_{i} x_{i}\right), \\
\mathbf{k}_{j}(t+1)= & \mathbf{k}_{j}(t)+v \cdot \sum_{\mathbf{k}} c_{i j} \cdot\left(\frac{y_{\mathbf{k}}^{\prime}-y_{\mathbf{k}}}{1+\left(y_{\mathbf{k}}^{\prime}-y_{\mathbf{k}}\right)^{2}}\right) \\
& \cdot \frac{x_{i}}{\tanh \left(\sum_{i} \omega_{j \mathbf{k}} x_{i}\right)} .
\end{aligned}
$$

In this paper, learning rate $v$ is considered as the adaptive scheme, in which its main idea is that the initial value of $v$ being set higher, generally about $0.7-0.9$, with the increase or decrement of the number of learning, may change in a law. When $v$ decreases to a certain extent, if $v$ has not still become convergence or error has still no improvement, $v$ is set again at about 0.5-0.7. $v$ enters the learning process again, until the end of the run. The learning rate is associated with the error function: when the error is reduced, increase the learning rate; when the error increases, reduce the learning rate:

$$
v_{(\mathbf{k})}= \begin{cases}1.12 v_{(\mathbf{k}-1)}, & E_{(\mathbf{k}-1)}>E_{(\mathbf{k})}, \\ 0.8 v_{(\mathbf{k}-1)}, & E_{(\mathbf{k}-1)}<E_{(\mathbf{k})}, \\ v_{(\mathbf{k}-1)}, & E_{(\mathbf{k}-1)}=E_{(\mathbf{k})} .\end{cases}
$$

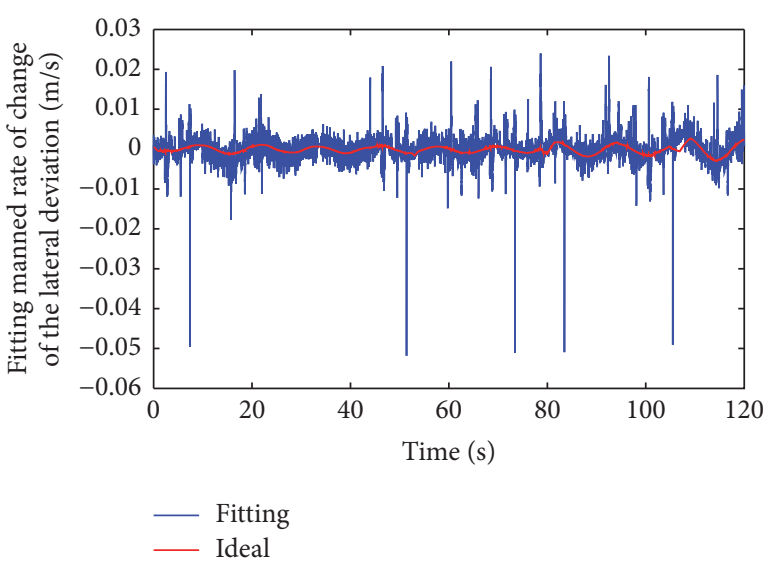

Figure 9: Fitting of change rate of the lateral deviation.

Identify the model parameters after obtaining the data after 6000 steps of learning:

$$
\begin{aligned}
& \mathbf{A}=\left[\begin{array}{ll}
0.0012 & 0.0996 \\
0.0095 & -0.0999
\end{array}\right], \\
& \mathbf{B}=\left[\begin{array}{c}
-0.007 \\
0.0803
\end{array}\right], \\
& \mathbf{k}=\left[\begin{array}{ccc}
1.000 & \\
& 1.009 & \\
& & 0.9998
\end{array}\right] .
\end{aligned}
$$

Then

$$
\begin{aligned}
{\left[\begin{array}{l}
\dot{x}_{1} \\
\dot{x}_{2}
\end{array}\right]=} & {\left[\begin{array}{ll}
0.0012 & 0.0996 \\
0.0095 & -0.0999
\end{array}\right]\left[\begin{array}{l}
\tanh \left(x_{1}\right) \\
\tanh \left(x_{2}\right)
\end{array}\right] } \\
& +\left[\begin{array}{c}
-0.007 \\
0.0803
\end{array}\right] \gamma .
\end{aligned}
$$

The fitting results of Figures 9 and 10 substantially eliminate the effect of outliers with relative error of control in less than $1 \%$ and less than $10 \%$. The trend is essentially coincident with the sample data.

The existing experimental data can be applied to the above-described method for fuzzy hyperbolic model parameter optimization in order to be close to the actual model. It provides the basis to the next controller design.

\section{Controller Design}

Based on this model, we can design conventional linear controllers or other nonlinear controllers. In this paper the controller is designed with hyperbolic tangent function of the state variables, so we can use language to describe the information of the controller. Thus this given controller is a fuzzy one [16]. 


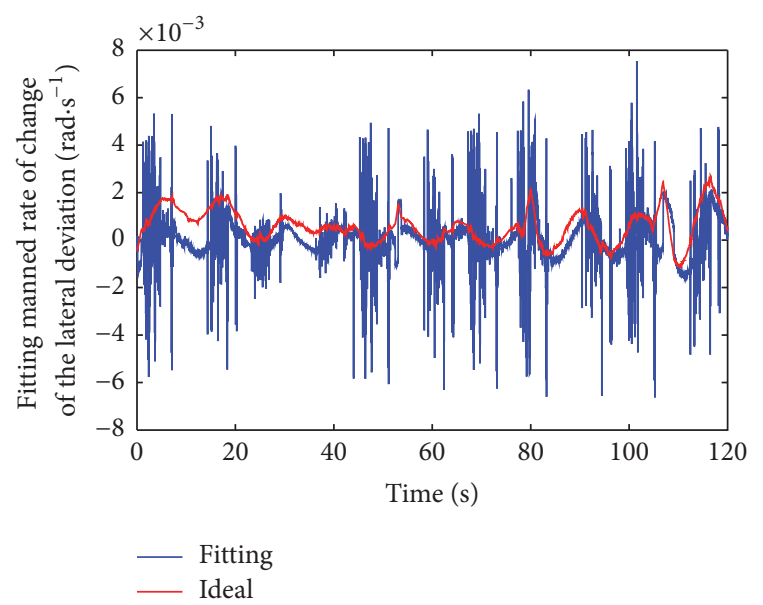

Figure 10: Fitting of change rate of the heading deviation.

The system can be stabilized by nonquadratic performance index function [16]. System performance can get to a minimum with $\mathbf{u}$ through the given $\mathbf{Q}$ and $\mathbf{R}$

$$
\dot{x}=\mathbf{A} f(x)+\mathbf{B u} .
$$

Definition 1 (see [17]). Let $S_{c}$ satisfy the following conditions: all sets of $f(\cdot)$ include $\mathbf{R} \rightarrow \mathbf{R}$ :

(1) $f$ is continuous;

(2) $f(0)=0$, for other $x \in \mathbf{R}, f(x) x>0$;

(3) when $|x| \rightarrow \infty, \int_{0}^{x} f(y) d y \rightarrow \infty$.

Theorem 2 (see [17]). For nonlinear system,

$$
\dot{x}=\mathbf{A} f(x)+\mathbf{B u}+\mathbf{D} w,
$$

where $f(x)=\left(f_{1}(x), \ldots, f_{n}(x)\right)^{\mathrm{T}}$ and $f(i) \in S_{c}$, and then $\mathbf{k}>0, \tanh (\mathbf{k} x) \in S_{c}(i=1,2,3, \ldots, n)$, and a performance index function can be proposed as

$$
\begin{aligned}
& J\left(x_{0}, t_{0}, \mathbf{u}\right)=\int_{t_{0}}^{\infty} f^{\mathrm{T}}(x(t)) \mathbf{Q} f(x(t)) \\
& +\mathbf{u}^{\mathrm{T}}(x(t)) \mathbf{R} \mathbf{u}(x(t))-w^{\mathrm{T}}(t) \mathbf{S} w(t) d t,
\end{aligned}
$$

where $\mathbf{Q}, \mathbf{R}$, and $\mathbf{S}$ are given as the definite positive symmetric constant matrix.

Design Optimal Control Vector [18]. Consider $\mathbf{u}^{*}(t)=\mathbf{u}^{*}(t)(t$, $\left.\{x(\tau)\}_{\tau=0}^{t}\right)$, and there is $\overline{\mathbf{L}}\left(x_{0}\right)$ with boundary. Then,

$$
\sup J\left(\mathbf{u}^{*}(t), w\right) \leq \overline{\mathbf{L}}\left(x_{0}\right) .
$$

If there is a diagonal positive definite matrix $\mathbf{P}_{\min }$ satisfying Riccati equation

$$
\mathbf{P A}+\mathbf{A}^{\mathrm{T}} \mathbf{P}-\mathbf{P}\left(\mathbf{B R}^{-1} \mathbf{B}^{\mathrm{T}} \mathbf{P}-\mathbf{D S}^{-1} \mathbf{D}^{\mathrm{T}}\right) \mathbf{P}+\mathbf{Q}=0,
$$

then $[A, B, \mathbf{Q}, \mathbf{R}]$ is optimized diagonal matrix.
Given nonlinear system and nonlinear quadratic performance index function, $[A, B, \mathbf{Q}, \mathbf{R}]$ is an optimized diagonal matrix. The optimal control vector is

$$
\mathbf{u}^{*}(t)=-\mathbf{R}^{-1} \mathbf{B}^{\mathrm{T}} \mathbf{P}_{\min } f(x(t)),
$$

where $\mathbf{P}_{\min }=\operatorname{diag}\left(p_{1}, \ldots, p_{n}\right)$ is optimized diagonal matrix. Let $\overline{\mathbf{L}}\left(x_{0}\right)=2 \sum_{i=1}^{n} p_{i} \int_{0}^{x_{i}\left(t_{0}\right)} f_{i}(\tau) d \tau$.

Thus, $\tanh (\mathbf{k} x) \in S_{c}$, and for fuzzy hyperbolic tangent, the quadratic performance index function is

$$
\begin{gathered}
J\left(x_{0}, t_{0}, \mathbf{u}\right)=\int_{t_{0}}^{\infty} \tanh ^{\mathrm{T}}(x(t)) \mathbf{Q} \tanh (x(t)) \\
+\mathbf{u}^{\mathrm{T}}(x(t)) \mathbf{R} \mathbf{u}(x(t))-w^{\mathrm{T}}(t) \mathbf{S} w(t) d t .
\end{gathered}
$$

Let $\mathbf{Q}=\left[\begin{array}{cc}10 & 0 \\ 0 & 15\end{array}\right], \mathbf{R}=0.8$, and $\mathbf{S}=800$ with Riccati equation; then

$$
\mathbf{P}=\left[\begin{array}{cc}
109.72 & 27.59 \\
27.59 & 16.78
\end{array}\right]
$$

According to the closed-loop system equation of state

$$
\dot{x}=\left(\mathbf{A}-\mathbf{B R}^{-1} \mathbf{B}^{\mathrm{T}} \mathbf{P}_{\text {min }}\right) f(x(t)) .
$$

Based on Lyapunov theorem and Riccati equation for this equation:

$$
\dot{x}=\left(\mathbf{A}-\mathbf{B R}^{-1} \mathbf{B}^{\mathrm{T}} \mathbf{P}_{\text {min }}\right) f(x(t)) .
$$

It is easy to prove

$$
\begin{gathered}
\mathbf{P}_{\text {min }}\left(\mathbf{A}-\mathbf{B R}^{-1} \mathbf{B}^{\mathrm{T}} \mathbf{P}_{\text {min }}\right)+\left(\mathbf{A}-\mathbf{B R}^{-1} \mathbf{B}^{\mathrm{T}} \mathbf{P}_{\text {min }}\right) \mathbf{P}_{\text {min }} \\
=-\left(\mathbf{Q}+\mathbf{P}_{\text {min }}\left(\mathbf{B} \mathbf{R}^{-1} \mathbf{B}^{\mathrm{T}} \mathbf{P}-\mathbf{D} \mathbf{S}^{-1} \mathbf{D}^{\mathrm{T}}\right) \mathbf{P}_{\text {min }}\right) .
\end{gathered}
$$

And $\left(\mathbf{A}-\mathbf{B R}^{-1} \mathbf{B}^{\mathrm{T}} \mathbf{P}\right)$ is diagonally stable; thus the closedloop system is asymptotically stable.

Then

$$
\mathbf{K}=\left[\begin{array}{ll}
11.627 & 6.135
\end{array}\right] \text {. }
$$

\section{HIL Simulation}

The difference between simulation and hardware-in-the-loop simulation is that there is a real-time simulation of the circuit's physical hardware. Hardware-in-the-loop simulation is intended to provide real-time incentives as the true signal to the controller so that the controller is connected to its real accused equipment and test its performance. Figure 11 is a pictorial diagram of the hardware simulation platform, in which c-RIO controller is recognized as the path tracking angle controller program compiled by the Simulink. PXI platform simulation runs the Adams, which can build underground mining articulated vehicle model, as the object of emulation. PC can run the LabVIEW graphical user interface for PXI and c-RIO real-time and display the data of simulation compiled from the process. Speed can be achieved by PID control at 


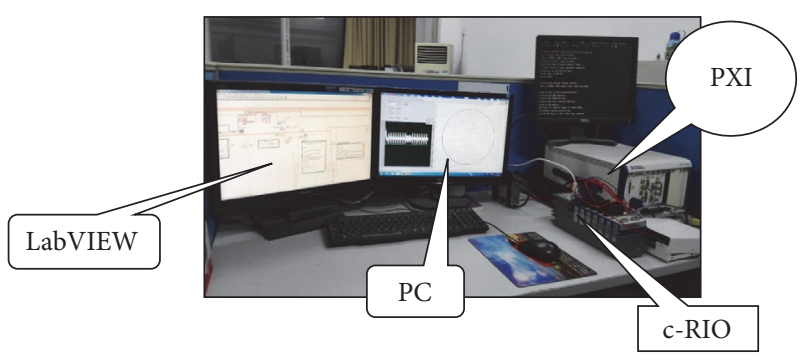

FIGURE 11: HIL simulation platform.

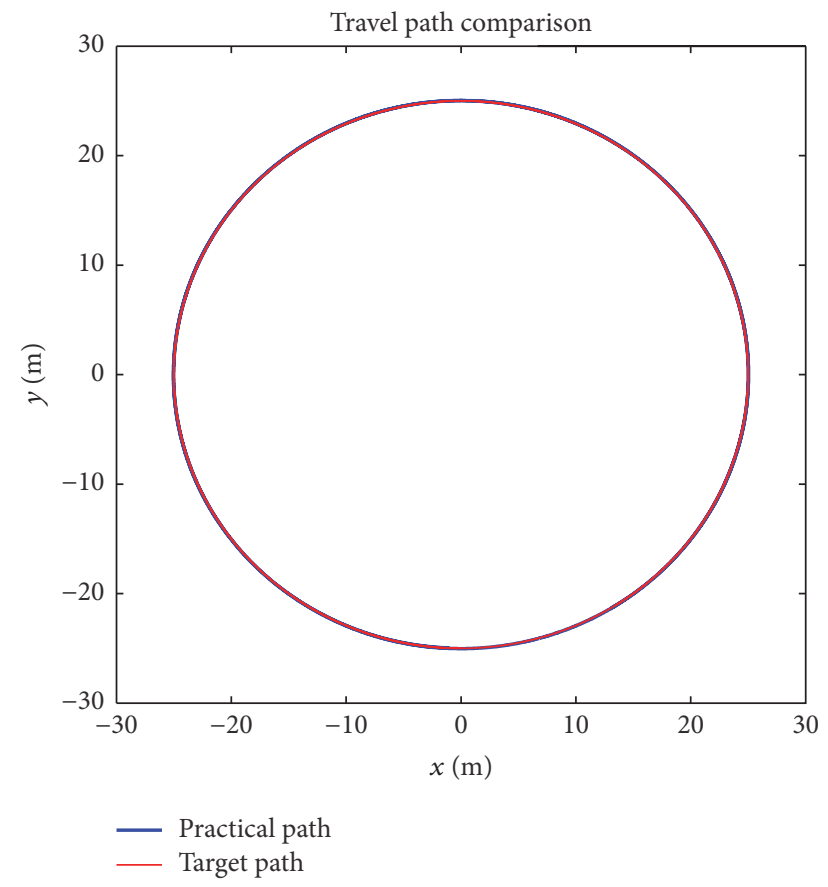

FIGURE 12: Simulation of driving route compared with the ideal path.

$v=3 \mathrm{~m} / \mathrm{s}$. So as to test this robust of control, there is one pit per $20 \mathrm{~m}$ on the road. The reference trajectory is set as a round whose center is $(0,0)$, and $\mathbf{R}$ is $25 \mathrm{~m}$ and an initial parameter setting is simulation time $200 \mathrm{~s}$, and the starting point coordinates $(-3,-25)$.

Derived from the simulation results, Figure 12 is the comparison chart of the actual trajectory and the reference trajectory. As can be seen from the simulation curve, the articulated vehicle traveling trace and the reference circular path are consistent, and the trajectory is relatively smooth. The speed which is controlled by PID from Figure 13 becomes finally steady at 30 seconds. The lateral displacement error is gradually flat from the beginning of violent shaking in Figure 14 . The overshoot amount is $4.28 \%$, and it is at most about $0.024 \mathrm{~m}$ at 18 seconds, with respect to the tread based on $2.280 \mathrm{~m}$, and the error is $1.7 \%$ and then close to the origin step by step. The orientation error is gradually flat from the beginning of violent shaking in Figure 15. The overshoot amount is $4.9 \%$, and it is at the top at about $0.08 \mathrm{rad}$ at 18 seconds and then close to the origin step by step. The articulated angle is gradually flat from the beginning of violent shaking in

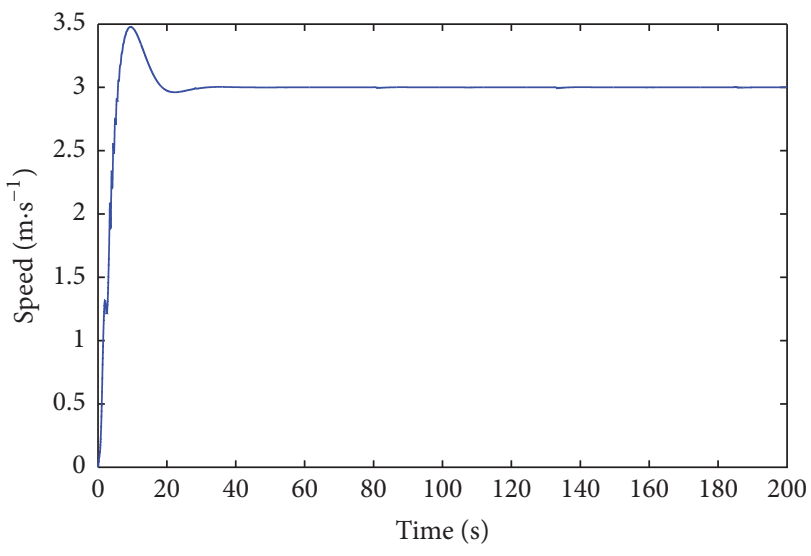

FIGURE 13: Longitudinal velocity of midpoint of front axle.

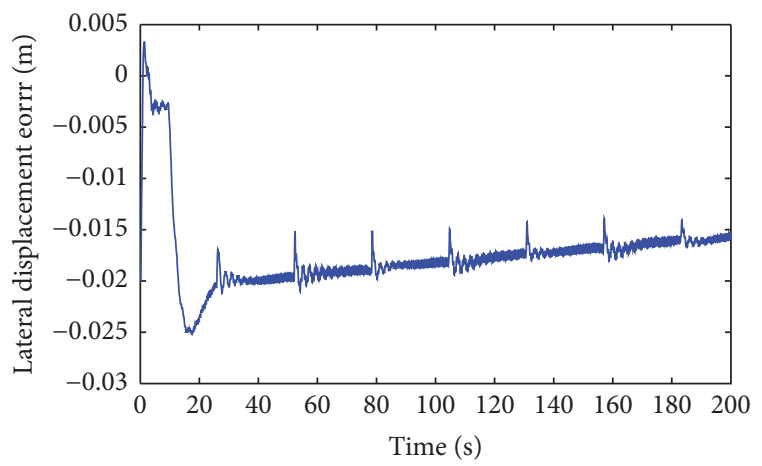

FIGURE 14: Lateral displacement error.

Figure 16. The overshoot amount is 3.5\%, and it is eventually stabilized at $0.21 \mathrm{rad}$ at 22 seconds. Compared with the articulated vehicle steering based on angle $45^{\circ}$, the error is $1.2 \%$. And the peaks are caused by pits, but then the system immediately recovers to the right path. It has better robustness than literature [12], in which there are a lot of jitters to adapt to the path as a similar situation. The results of literature [10] are as follows: lateral deviation was $1 \mathrm{~m}$ and heading angle deviation is $0.1 \mathrm{rad}$. Literature [19] also analyzed the models of articulated vehicle to control it with synovial control method, whose results of the simulation are as follows: the lateral deviation is $0.1 \mathrm{~m}$ and heading angle deviation was $0.17 \mathrm{rad}$. So there exists a large gap when compared with the results of this paper.

\section{Conclusions}

This article provides a method used in unmanned systems based on the way of fuzzy hyperbolic pole control act for articulated vehicle trajectory tracking accurately. Conclusions are as follows.

(1) Fuzzy hyperbolic tangent model can well describe the quantitative relation among lateral displacement error, the orientation error, and the articulated angle.

(2) Based on the method of Cauchy robust error estimator, the weights of BP neural network can effectively 


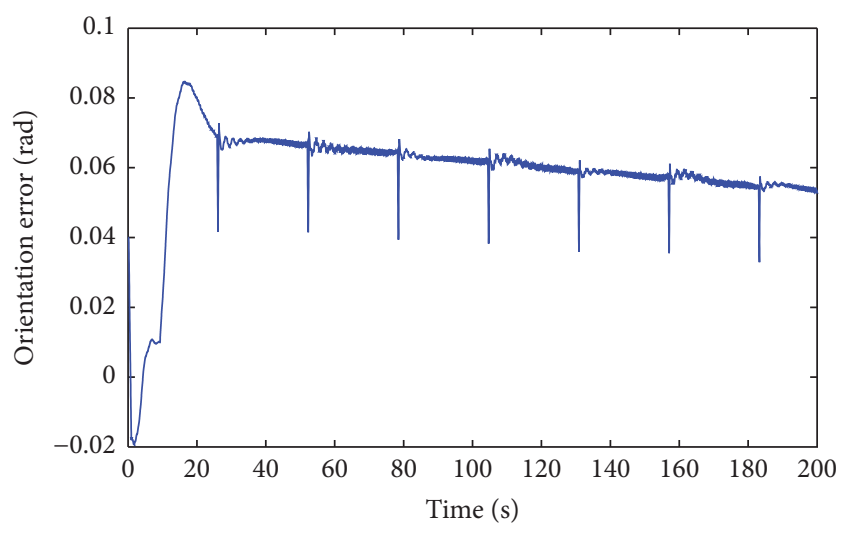

FIGURE 15: Unmanned orientation error.

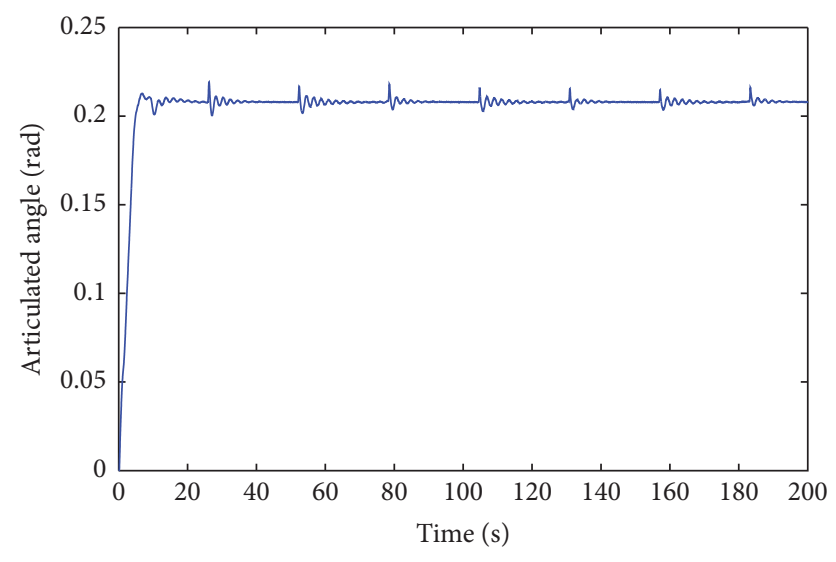

Figure 16: Articulated angle.

be reduced by the influence of singular error of neural network learning and fitting error, and relative error below expectations can be decreased so that it can achieve the system of identification.

(3) By the $H$-infinity controller design, controller has a good control performance, and it makes the system keep a better performance about robustness in order to achieve the purpose of the comprehensively optimal control error.

(4) This method can be used in articulated vehicle path tracking effectively based on the $H$-infinity controller. Simulation in hardware-in-the-loop shows that overshoot and the response time are less than expectations and are eventually to stabilize. The controller has met the requirement of the real-time control performance.

\section{Competing Interests}

Guangsi Shi, Jue Yang, Xuan Zhao, Yanfeng Li, Yalun Zhao, and Jian Li declare that there is no conflict of interests regarding the publication of this manuscript.

\section{Acknowledgments}

This work was financially supported by the National High Technology Research and Development Program (863 Program) of China, under Award 2011AA060404 Intelligent Underground Mining Truck, and Fundamental Research Funds for the Central Universities, FRF-TP-16-004A1, research on path-planning and path-following algorithm for underground mining vehicles based on reinforcement learning.

\section{References}

[1] X.-J. Zeng and M. G. Singh, "Approximation theory of fuzzy systems-SISO case," IEEE Transactions on Fuzzy Systems, vol. 2, no. 2, pp. 162-176, 1994.

[2] L. X. Wang, "Fuzzy systems are universal approximations," in Proceeding of the IEEE International Conference on Fuzzy Systems, pp. 1163-1170, 1992.

[3] T. Takagi and M. Sugeno, "Fuzzy identification of systems and its applications to modeling and control," IEEE Transactions on Systems, Man and Cybernetics, vol. 15, no. 1, pp. 116-132, 1985.

[4] M. Sugeno and T. Yasukawa, "Fuzzy-logic-based approach to qualitative modeling," IEEE Transactions on Fuzzy Systems, vol. 1, no. 1, pp. 7-31, 1993.

[5] S. Scheding, G. Dissanayake, E. Nebot, and H. Durrant-Whyte, "Slip modelling and aided inertial navigation of an LHD," in Proceedings of the IEEE International Conference on Robotics and Automation, pp. 1904-1909, Institute of Electrical and Electronics Engineers, Albuquerque, NM, USA, April 1997.

[6] H. Zhang and Y. Quan, "Modeling, identification, and control of a class of nonlinear systems," IEEE Transactions on Fuzzy Systems, vol. 9, no. 2, pp. 349-354, 2001.

[7] T. Nayl, G. Nikolakopoulos, and T. Gustfsson, "Switching model predictive control for an articulated vehicle under varying slip angle," in Proceedings of the 20th Mediterranean Conference on Control \& Automation (MED '12), pp. 890-895, IEEE Computer Society, Barcelona, Spain, July 2012.

[8] J. H. Lee and W. S. Yoo, "Predictive control of a vehicle trajectory using a coupled vector with vehicle velocity and sideslip angle," International Journal of Automotive Technology, vol. 10, no. 2, pp. 211-217, 2009.

[9] P. Ridley and P. Corke, "Load haul dump vehicle kinematics and control," Journal of Dynamic Systems, Measurement, and Control, vol. 125, no. 1, pp. 54-59, 2003.

[10] Z. Xuan, Y. Jue, and Z. Wenming, "Feedback linearization control for path tracking of articulated dump truck," TELKOMNIKA Telecommunication, Computing, Electronics and Control, vol. 9, no. 13, pp. 922-929, 2015.

[11] D. Piyabongkarn, R. Rajamani, J. A. Grogg, and J. Y. Lew, "Development and experimental evaluation of a slip angle estimator for vehicle stability control," IEEE Transactions on Control Systems Technology, vol. 17, no. 1, pp. 78-88, 2009.

[12] Y. Qi, Trajectory Control for the Underground Articulated Dump Truck Based on Model Predictive Control, University of Science and Technology Beijing, Beijing, China, 2013.

[13] K.-K. D. Young, "Controller design for a manipulator using theory of variable structure systems," IEEE Transactions on Systems, Man and Cybernetics, vol. 8, no. 2, pp. 101-109, 1978. 
[14] E. M. Jafarov, M. N. A. Parlakçi, and Y. Istefanopulos, "A new variable structure PID-controller design for robot manipulators," IEEE Transactions on Control Systems Technology, vol. 13, no. 1, pp. 122-130, 2005.

[15] P. Petrov and P. Bigras, "A practical approach to feedback path control for an articulated mining vehicle," in Proceedings of the IEEE/RSJ International Conference on Intelligent Robots and Systems, pp. 2258-2263, Institute of Electrical and Electronics Engineers, Maui, Hawaii, USA, November 2001.

[16] G.-T. Hui, H.-G. Zhang, G. Wang, X.-P. Xie, and Z.-N. Wu, "Research on fuzzy hyperbolic tangent model: a review," Acta Automatica Sinica, vol. 39, no. 11, pp. 1849-1857, 2013.

[17] E. Kaszkurewicz and A. Bhaya, "Robust stability and diagonal Liapunov functions," SIAM Journal on Matrix Analysis and Applications, vol. 14, no. 2, pp. 508-520, 1993.

[18] K. Ogata, Modern Control Engineering, Electronic Industry Press, Beijing, China, 2011.

[19] X. Zhao, J. Yang, W. Zhang, and J. Zeng, "Sliding mode control algorithm for path tracking of articulated dump truck," Transactions of the Chinese Society of Agricultural Engineering, vol. 31, no. 10, pp. 198-203, 2015. 


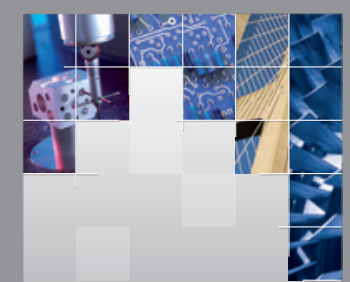

\section{Enfincering}
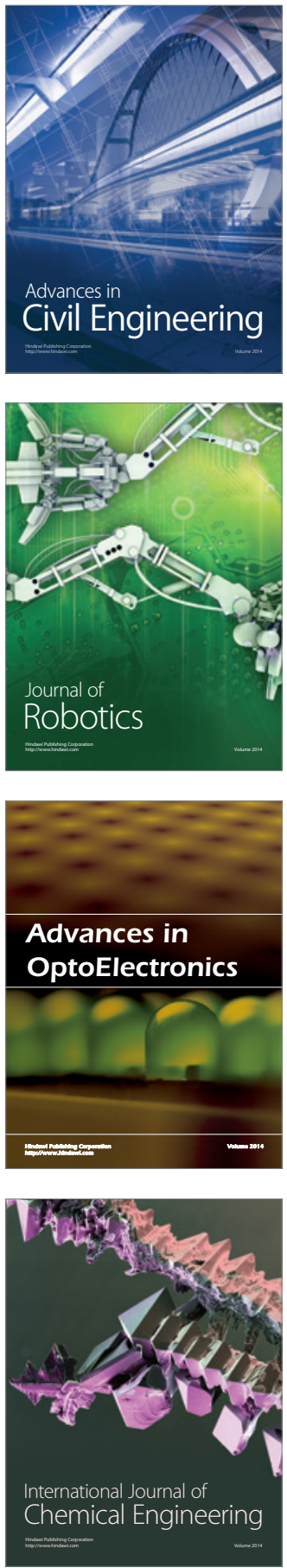

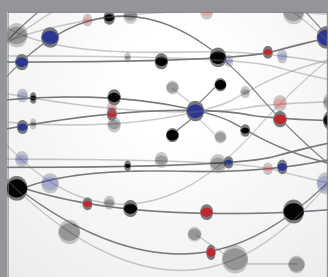

The Scientific World Journal

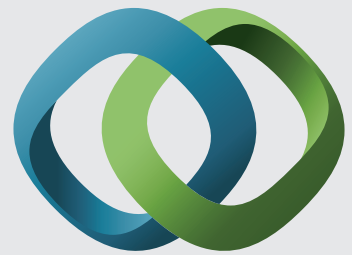

\section{Hindawi}

Submit your manuscripts at

http://www.hindawi.com
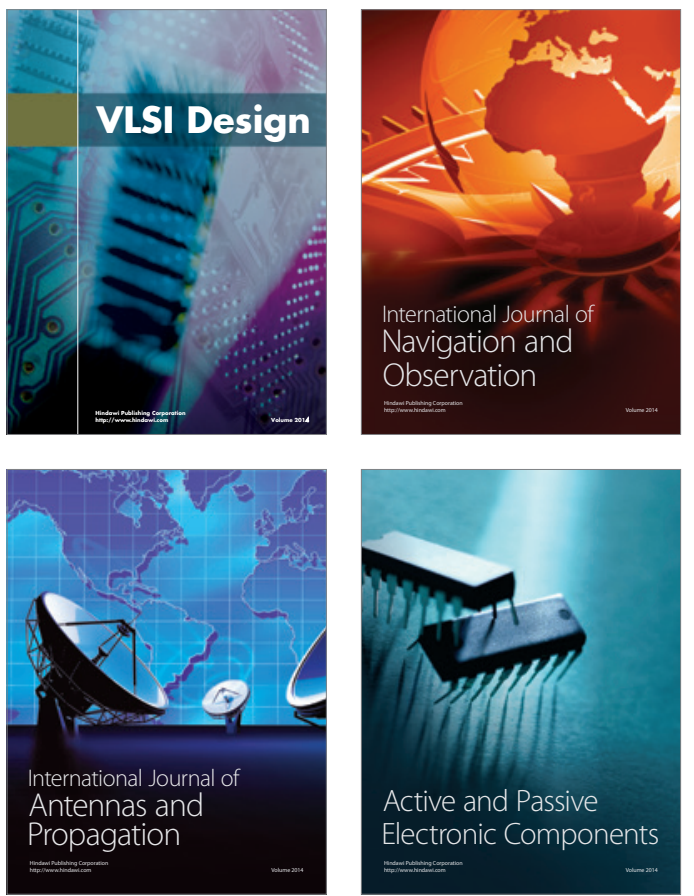
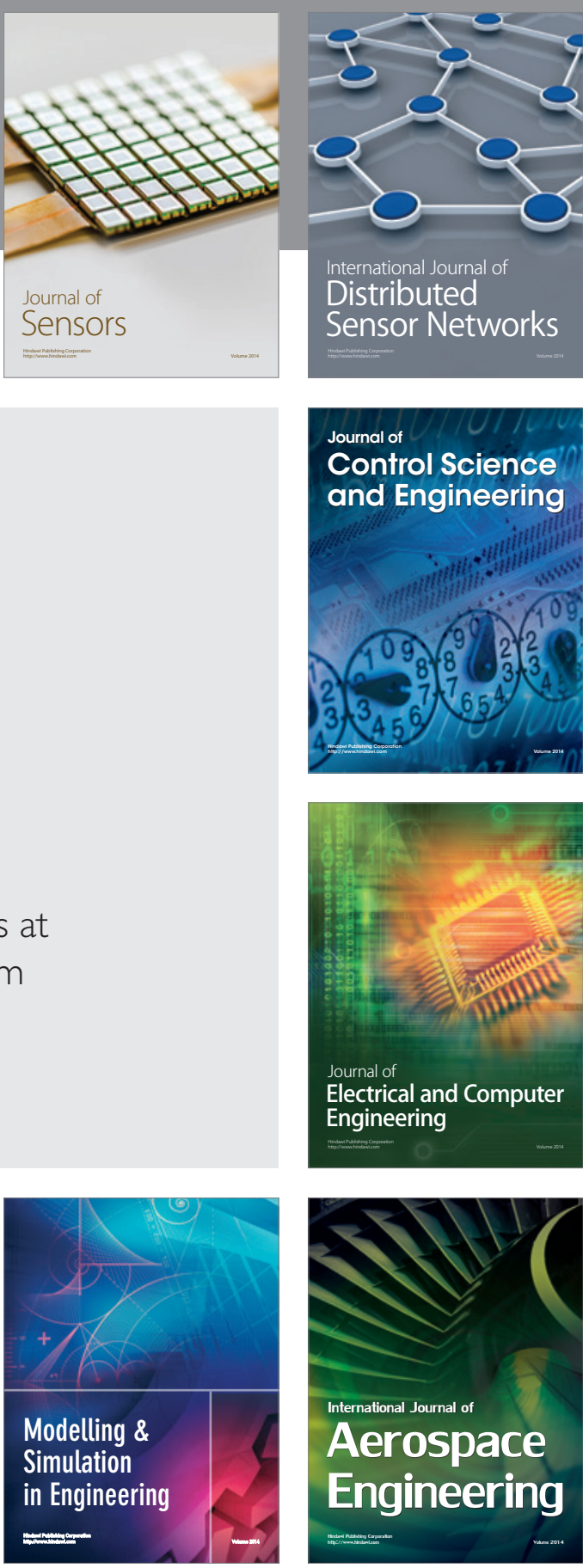

International Journal of

Distributed

Sensor Networks

Journal of

Control Science

and Engineering
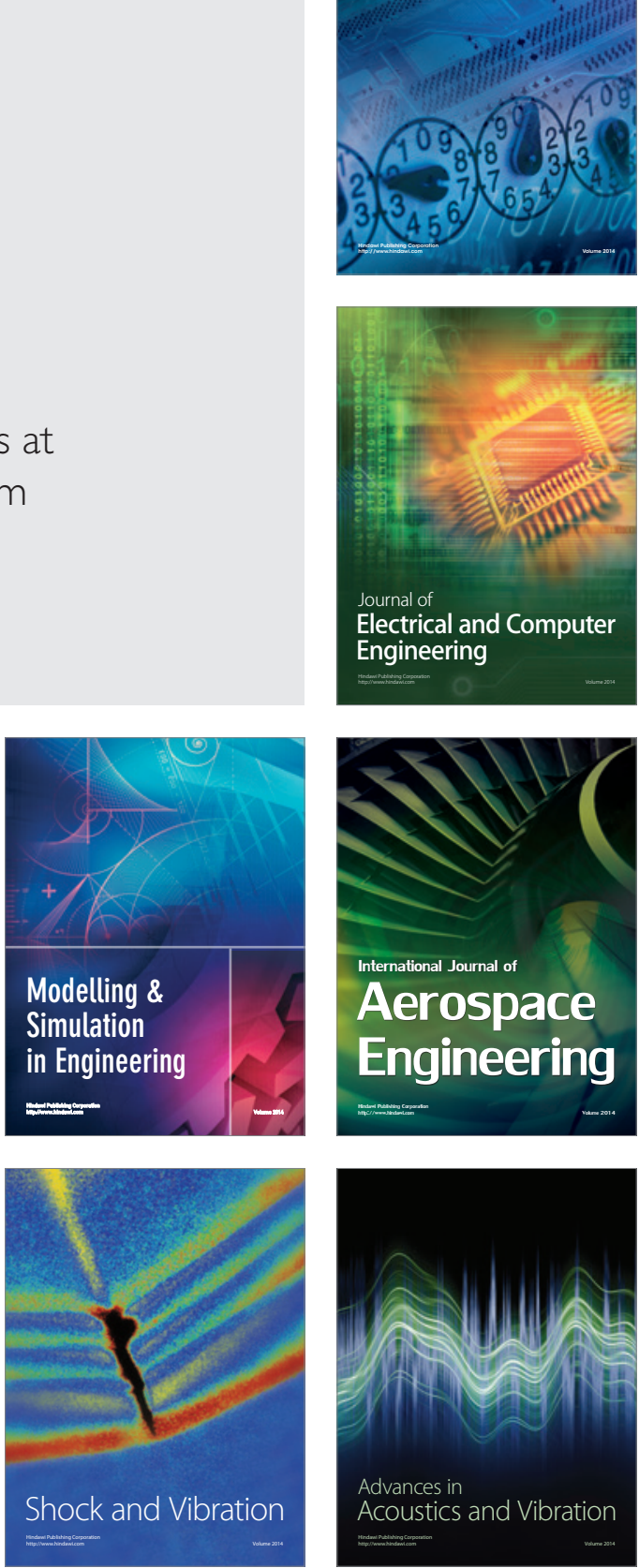